\title{
LA COBERTURA DE LOS ACCIDENTES DEL TRABAJO Y ENFERMEDADES PROFESIONALES POR LAS NORMAS INTERNACIONALES DEL TRABAJO DE LA OIT
}

\author{
Dr. Pablo Arellano OrTiz* \\ Universidad Austral de Chile
}

\begin{abstract}
RESUMEN: El presente trabajo pretende pasar revista a los principales instrumentos internacionales, emanados de la Organización Internacional del Trabajo (OIT), que guardan relación con la protección de los accidentes del trabajo y enfermedades profesionales. Además, detalla las contingencias cubiertas por cada uno de los Convenios estudiados; la ratificación y denuncia de los mismos.
\end{abstract}

Palabras clave: Accidentes del trabajo y enfermedades profesionales, normas internacionales del trabajo, Organización Internacional del Trabajo (OIT), seguridad social.

ABSTRACT: The study aims to do a review of the major international instruments emanating from the International Labor Organization (ILO), which relate to the protection of employment injury. In addition, it details the contingencies covered by each of the conventions studied, ratification and denunciation of them.

Key words: Employment injury, international labour standards, International Labor Organization (ILO), social security.

\section{INTRODUCCIÓN}

Las Normas Internacionales del Trabajo que emanan de la Organización Internacional del Trabajo (OIT) buscan establecer un marco mínimo en las legislaciones de los países miembros en vista de una protección a través de los derechos de los trabajadores de sus países. De esta manera la OIT siempre ha otorgado una gran importancia a la seguridad social en conformidad con su mandato constitucional ${ }^{1}$ que consiste, entre otras cosas, en mejorar las condiciones del trabajo a través de "la lucha contra el desempleo,... la protección del trabajador contra las enfermedades, sean o no profesionales, y contra los accidentes de trabajo,... las pensiones de vejez e invalidez" 2 . En particular, en lo relativo a la protección del trabajador contra las enfermedades, sean o no profesionales y contra los accidentes de trabajo, ha estado siempre en primera línea de las preocupaciones de la OIT: figura en el Preámbulo de su Constitución, y se reafirma en la Declaración de Filadelfia que "reconoce la obligación

\footnotetext{
Profesor de Derecho del Trabajo y de Seguridad Social, Universidad Austral de Chile. Doctor en Derecho de la Université Paris Ouest Nanterre La Defense, Francia; Master 2 Recherche Droit Social et Droit de la Santé de la Université de Paris X Nanterre, Francia; Master 2 Recherche Droit Social de la Université de Paris II Panthéon Assas, Francia. Abogado, Universidad de Concepción, Chile. Ha trabajado como consultor internacional en temas de seguridad social y también se ha desempeñado como jurista en el Departamento de Normas de la Organización Internacional del Trabajo. Además, ha publicado diversos artículos en español y en inglés sobre temas relacionados con el derecho a la seguridad social y sobre normas internacionales del trabajo, particularmente en relación con desempleo, pensiones y salud, sobre legislación chilena e internacional.

1 Sobre este punto se puede profundizar nuestra posición en: ARELLANO ORTIZ, Pablo, "Reflexiones sobre las evoluciones recientes en materia de protección de riesgos sociales a nivel internacional”, en: Revista de Derecho Universidad Católica del Norte, vol.17, No 2, 2010, pp.167-183.

2 Constitución de la Organización International del Trabajo, Tratado de Versailles, Parte XIII, 1919.
} 
solemne de la Organización Internacional del Trabajo de fomentar, entre todas las naciones del mundo, programas que permitan: [...] g) proteger adecuadamente la vida y la salud de los trabajadores en todas las ocupaciones..."3.

En junio de 2001, la Conferencia realizó una discusión general con el objetivo de establecer una visión de la OIT sobre la seguridad social al alba del siglo XXI. Se llegó a la conclusión de que las actividades de la OIT en materia de seguridad social debían basarse en la declaración de Filadelfia, el concepto de trabajo decente ${ }^{4}$ y las normas pertinentes en el campo de la seguridad social ${ }^{5}$.

Paralelamente, el Consejo de Administración llevó a cabo, entre 1995 y 2002, un trabajo de evaluación del conjunto de las normas de la OIT. En el marco de este examen, llegó a la conclusión de que el Convenio $\mathrm{N}^{\circ} 102$, así como los convenios y recomendaciones adoptados posteriormente en el ámbito de la seguridad social se encuentran actualizados y, por lo tanto, la oficina debe promocionar su ratificación ${ }^{6}$. En el Anexo 1 hemos incluido las normas de la OIT en materia de accidentes del trabajo y enfermedades profesionales que se encuentran al día y aquellas consideradas como ya superadas. Como veremos, solo algunas de estas normas están actualizadas y deben ser objeto de actividades de promoción por parte de la OIT. Las normas más recientes en este ámbito fueron adoptadas en junio de $2000 \mathrm{y}$ tratan de la protección de la maternidad ${ }^{7}$.

En general, el conjunto de estos instrumentos de la OIT relativos a la seguridad social se dividen en tres "generaciones" de normas, que corresponden a acercamientos distintos ${ }^{8}$. Las normas de la primera generación corresponden a aquellas adoptadas hasta fines

\footnotetext{
La Declaración relativa a los fines y objetivos de la Organización Internacional del Trabajo, adoptada en la Conferencia Internacional del Trabajo en su 22a sesión, el 10 de mayo de 1944, en Filadelfia, se encuentra anexada a la Constitución de la organización y forma parte de la misma.

4 La Agenda de Trabajo Decente aparece definida como tal en 1999, presentada por el Director de la OIT, Juan Somavía, en la 87a Sesión de la Conferencia Internacional del Trabajo. Este "concepto", que recoge de una forma novedosa y concreta los objetivos que la OIT viene persiguiendo desde su constitución hace más de 90 años, ha sido consensuado por los tres mandantes de la Organización: gobiernos, organizaciones empresariales y organizaciones sindicales. "El trabajo decente resume las aspiraciones de las personas en su vida laboral, aspiraciones en relación a oportunidades e ingresos; derechos, voz y reconocimiento; estabilidad familiar y desarrollo personal; justicia e igualdad de género. Las diversas dimensiones del trabajo decente son pilares de la paz en las comunidades y en la sociedad. El trabajo decente refleja las preocupaciones de gobiernos, trabajadores y empleadores, que dan a la OIT su singular identidad tripartita. El trabajo decente puede ser sintetizado en cuatro objetivos estratégicos: principios y derechos fundamentales en el trabajo y normas laborales internacionales; oportunidades de empleo e ingresos; protección y seguridad social; y diálogo social y tripartismo. Estos objetivos tienen validez para todos los trabajadores, mujeres y hombres, en la economía formal e informal, en trabajos asalariados o autónomos; en el campo, industria y oficina; en sus casas o en la comunidad" ver: http://www.ilo.org/public/spanish/standards/relm/ilc/ilc87/rep-i.htm y OIT. Trabajo Decente, Memoria del Director General, Conferencia Internacional del Trabajo, 87a reunión. Ginebra, 1999.

OIT. Seguridad social: un nuevo consenso. Ginebra, 2002.

6 En el año 2002, el Consejo de Administración de la OIT ha confirmado que 8 de los 31 convenios existentes sobre seguridad social se encontraban al día. A saber: Convenio No 102 sobre la seguridad social (norma mínima), de 1952; Convenio No 118 sobre la igualdad de trato (seguridad social), de 1962; Convenio No 121 sobre las prestaciones en caso de accidentes del trabajo y enfermedades profesionales, de 1964; Convenio No 128 sobre las prestaciones de invalidez, vejez y sobrevivientes, de 1967; Convenio No 130 sobre asistencia médica y prestaciones monetarias de enfermedad, de 1969; Convenio No 157 sobre la conservación de los derechos en materia de seguridad social, de 1982; Convenio No 168 sobre el fomento del empleo y la protección contra el desempleo, de 1988; y, Convenio № 183 sobre la protección de la maternidad, de 2000.

7 Convenio $\mathrm{N}^{\circ} 183$ sobre la protección de la maternidad, de 2000.

8 Ver: Humblet, M.; Silva, R, Normas para el siglo XXI, Seguridad Social. Ginebra: OIT, 2002.
} 
de la Segunda Guerra Mundial y la mayor parte de ellas se basaban en el seguro social como mecanismo de protección. Sin embargo, los convenios relativos a la indemnización de las lesiones profesionales aceptaban los sistemas de protección basados en la responsabilidad del empleador. En este período las normas se refieren, en particular, al establecimiento de un sistema de seguro obligatorio relativo a un riesgo determinado que cubra los principales sectores de actividad y las principales categorías de trabajadores. Las primeras normas adoptadas abordaron las cuestiones que parecían más urgentes y que estaban en condiciones de ser objeto de una acción internacional (la maternidad, las lesiones profesionales, la enfermedad, etc.). En definitiva, estas normas solo se aplicaban a ciertas categorías de trabajadores y no al conjunto de la población. Cada uno de los instrumentos cubría un riesgo específico; además, para ciertos riesgos, se adoptaron instrumentos distintos para diferentes sectores de actividad (industria y agricultura, entre otros).

La segunda generación de normas corresponde a la era de la seguridad social, al nacimiento de esta rama. La actividad normativa de la OIT se enfoca en una concepción global y más amplia de la protección. El objetivo es unificar y coordinar los diferentes regímenes de protección en un sistema único de seguridad social que cubra todas las contingencias y a todos los trabajadores. De esta manera se podría cumplir con el objetivo de la universalidad objetiva y subjetiva. Así, después de la Segunda Guerra Mundial, las normas se inspiraron en el concepto más general de seguridad social desarrollado en el Informe Beveridge. De esta forma la Declaración de Filadelfia, adoptada en 1944, redefinió los objetivos de la OIT, incluyendo la extensión de las medidas de seguridad social con vistas a garantizar unos ingresos básicos a todos los que necesitan dicha protección, así como asistencia médica completa. Es así como en la Conferencia de 1944 se adoptaron dos recomendaciones importantes que siguen esta concepción: la Recomendación No 67 sobre la seguridad de los medios de existencia, 1944, y la Recomendación No 69 sobre la asistencia médica, 1944. Esta concepción inspiró, asimismo, a la Conferencia cuando adoptó el Convenio No 102 sobre la seguridad social (norma mínima), en 1952. Como su título indica, este Convenio prevé un nivel mínimo de prestaciones para cada una de las nueve ramas de seguridad social que cubre. Teniendo en cuenta las cláusulas de flexibilidad del Convenio No 102, este nivel mínimo constituye un objetivo que los Estados Miembros de la OIT deberían alcanzar -o en el caso de ciertos Estados, superar- sea cual sea su nivel de desarrollo económico.

La tercera generación de normas de seguridad social corresponde a los instrumentos posteriores al Convenio No 102. Están redactados siguiendo el modelo del Convenio No 102, aunque ofrecen una protección superior en términos de población cubierta y de nivel de prestaciones. Cabe mencionar a este respecto que se había previsto adoptar, en forma paralela al Convenio No 102, un instrumento relativo a una norma superior de seguridad social $^{9}$. Por falta de tiempo y debido a su complejidad, la cuestión finalmente no fue tratada en la reunión de la Conferencia en $1952^{10}$. Sin embargo, la Conferencia invitó al Consejo de Administración a retomar la cuestión oportunamente. Por último, la idea de un instru-

\footnotetext{
9 Revisar: OIT, Objetivos y norma avanzada de la seguridad social. Informe V b). Quinto punto del orden del día. Conferencia Internacional del Trabajo. Trigésima quinta reunión-1952. Ginebra, 1952.

10 Revisar: OIT, Acta de las sesiones. Conferencia Internacional del Trabajo. Trigésima quinta reunión-1952. Ginebra, 1953.
} 
mento único fue dejada de lado y en cambio, se adoptaron progresivamente una serie de instrumentos para completar las normas anteriores ${ }^{11}$. De manera general, tales instrumentos revisan los convenios de la primera generación y van más allá, estableciendo un grado de protección más elevado que el previsto en el Convenio No 102 en el cual se establecen las normas mínimas.

Con el fin de comprender mejor la evolución de las normas de protección de los accidentes del trabajo y enfermedades profesionales, estructuraremos esta presentación en relación a las tres generaciones de normas en materia de seguridad social ya enunciadas.

\section{PRIMERAS NORMAS DE PROTECCIÓN}

La historia de la evolución de la protección de los accidentes del trabajo y de las enfermedades profesionales comenzó tímidamente en 1921 con el Convenio No 12 sobre indemnización por accidentes del trabajo (agricultura), el cual proveía simplemente la extensión a los trabajadores agrícolas la reparación de los accidentes del trabajo. Este convenio fue ratificado por Chile el 15 de septiembre de 1925 y todavía es vinculante para nuestra legislación, puesto que no ha sido denunciado.

Es con el Convenio No 17 sobre la indemnización por accidentes del trabajo, de 1925, que realmente se introducen reglas de fondo sobre el tema. Este Convenio se aplicó a las empresas, explotaciones o establecimientos de cualquier naturaleza, públicos o privados, estableciendo que las indemnizaciones debidas por ocasión de un accidente del trabajo en caso de fallecimiento o que tengan como consecuencia una incapacidad permanente eran pagadas a la víctima o a sus herederos en forma de una renta. Sin embargo, estas indemnizaciones podían ser pagadas en su totalidad o una parte en forma de un capital, siempre y cuando la garantía de un empleo juicioso fuera garantizada. Además, el convenio agregó una regla nueva para la época, exigiendo un complemento de indemnización para los casos en que las víctimas de los accidentes resultaran con incapacidad y necesitaran de una asistencia constante de otra persona. Se previó la adopción de medidas nacionales de control, así como también métodos de revisión de las indemnizaciones. También se consagró el derecho de las víctimas de accidentes a la asistencia quirúrgica y farmacéutica necesaria, así como también de prótesis y ortopedia. Contenía, a su vez, disposiciones con el fin de garantizar las prestaciones en caso de insolvencia del empleador o del asegurador. Nuestro país ratificó el Convenio $\mathrm{N}^{\circ} 17$ sobre la indemnización por accidentes del trabajo, de 1925, el 8 de octubre de 1931, sin embargo este convenio ya no es vinculante para nuestra legislación, puesto que fue denunciado el 8 de agosto de 2000.

Debemos añadir que el Convenio No 17 fue completado por dos recomendaciones, las No 22 y No 23. La primera trata el tema del monto mínimo de las indemnizaciones en materia de reparación de los accidentes del trabajo, fijándolo en $2 / 3$ del salario de la vícti-

\footnotetext{
11 Se trata del Convenio sobre igualdad de trato (seguridad social), de 1962 (No 118); el Convenio sobre prestaciones en caso de accidentes de trabajo y enfermedades profesionales, 1964 ( No 121) [Cuadro I modificado en 1980]; el Convenio sobre las prestaciones de vejez, invalidez y sobrevivientes, de 1967 ( No 128); el Convenio sobre asistencia médica y prestaciones monetarias de enfermedad, de 1969 (No 130); el Convenio sobre la conservación de los derechos en materia de seguridad social, de 1982 (No 157); el Convenio sobre el fomento del empleo y la protección contra el desempleo, de 1988 (No 168) y el Convenio sobre la protección de la maternidad, 2000 ( No 183), así como de las recomendaciones que acompañan a estos convenios.
} 
ma. También promueve que el suplemento de indemnización en caso de enfermedades que necesiten de la asistencia constante de otra persona debería ser, a lo menos, la mitad de la prestación otorgada en caso de incapacidad total permanente. Además, la recomendación enumera una lista de las personas que deberían ser considerados como herederos de la víctima en caso de accidente del trabajo seguido de fallecimiento. Por último, la recomendación prevé la reeducación profesional para las víctimas de accidentes del trabajo. Por su lado, la recomendación No 23 trata del tema de la jurisdicción competente para la resolución de los conflictos relativos a la reparación de los accidentes del trabajo.

El Convenio No 17 fue además completado por el Convenio No 19 que prevé una igualdad de trato en materia de accidentes del trabajo para los trabajadores extranjeros de los países que ratifiquen el convenio y para sus herederos. El Convenio No 19 sobre la igualdad de trato (accidentes del trabajo), de 1925, fue ratificado el 8 de octubre de 1931 y es actualmente vinculante para nuestro país.

Por su parte, en 1925, fue adoptado el Convenio No 18, el primero sobre enfermedades profesionales. Nuestro país lo ratificó el 31 de mayo de 1933, y lo denunció el 8 de agosto de 2000. Este Convenio incluyó por primera vez una lista de enfermedades consideradas de origen profesional, a saber: intoxicación por plomo, intoxicación por mercurio e infección carbonosa. Pero fue la Recomendación No 24, adoptada el mismo año, que solicitó a cada país instaurar un procedimiento simple para la revisión de la lista de enfermedades consideradas como profesionales según la legislación nacional. En 1934 este convenio fue revisado por el Convenio No 42 sobre enfermedades profesionales, que contenía una lista de 10 enfermedades profesionales, incluyendo en ella a la silicosis. Este convenio fue ratificado por Chile el 14 de octubre de 1994 para ser luego denunciado el 30 de septiembre de 1999. La lista de enfermedades es revisada, posteriormente, en 1964 cuando se adoptó el Convenio No 121 sobre las prestaciones en caso de accidentes del trabajo y enfermedades profesionales que, en un anexo, incluyó una lista de enfermedades profesionales. Dicho anexo fue modificado por la Conferencia Internacional del Trabajo en 1980. El Convenio No 18 y la Recomendación No 24 previó que la reparación de las enfermedades profesionales debe estar basada en principios generales de la legislación sobre la reparación de accidentes del trabajo y que la tasa de reemplazo de la reparación no debía ser inferior a aquella que la legislación previó para los daños resultantes de los accidentes del trabajo.

\section{LA APARICIÓN DE LA SEGURIDAD SOCIAL Y LA PROTECCIÓN DE LOS ACCIDENTES DEL TRABAJO Y DE LAS ENFERMEDADES PROFESIONALES}

Fue en la Conferencia Internacional del Trabajo de $1952^{12}$ donde se aprobó el convenio

\footnotetext{
$12 \mathrm{Al}$ respecto ver OIT, “Acta de... (n. 10). Además ver los trabajos preparatorios para su adopción: OIT, Norma minima de la seguridad social, Informe V a) (1). Quinto punto del orden del día, Conferencia Internacional del trabajo, Trigésima quinta reunión-1952. Ginebra, 1951; OIT, Norma minima de la seguridad social, Informe V a) (2). Quinto punto del orden del día, Conferencia Internacional del trabajo, Trigésima quinta reunión-1952, Ginebra, 1952; OIT, Objetivos y norma avanzada de la seguridad social, Informe V b). Quinto punto del orden del día, Conferencia Internacional del trabajo, Trigésima quinta reunión-1952, Ginebra, 1952; OIT, Objetivos y normas minimas de la seguridad social, Informe IV (1), Cuarto punto del orden del día, Conferencia Internacional del trabajo, Trigésima cuarta reunión-1951, Ginebra, 1950; OIT, La seguridad social: estudio internacional, Estudios y documentos, Nueva serie No 23, Ginebra, 1950; OIT, Objetivos y normas mínimas de la seguridad
} 
de base de la posguerra que, en el ámbito de la seguridad social, no solo inspiró el trabajo de la OIT, sino también el de otras organizaciones internacionales: el Convenio No 102 de Seguridad Social (norma mínima) ${ }^{13}$. Este texto, aún en vigor y considerado al día ${ }^{14}$, tiene por objetivo definir globalmente una norma de conjunto de la seguridad social que pueda llegar a todos los Estados, tomando en cuenta su situación particular. Los textos anteriores distinguen cada rama de la seguridad social y cada sector de actividad. El Convenio No 102 se basa en el principio del sistema general de seguridad, el cual engloba al conjunto de las eventualidades (estados de necesidad o riesgos sociales) y de las prestaciones, y se extiende gradualmente a toda la población. El Convenio aborda simultáneamente las nueve ramas/riesgos principales de la seguridad social: asistencia médica, prestaciones de enfermedad, prestaciones de desempleo, prestaciones de vejez, prestaciones en caso de accidentes del trabajo y enfermedades profesionales, prestaciones familiares, prestaciones por maternidad, invalidez y sobrevivientes. Un Estado, a fin de estar en condiciones de ratificar el Convenio ${ }^{15}$, debe comprometerse a cubrir al menos tres de estas ramas, y entre las tres ramas debe ser aceptada al menos una de ellas relativa a las prestaciones de desempleo, vejez, accidentes del trabajo y enfermedad, invalidez o sobrevivientes. Obviamente, puede comprometerse a cubrir otras ramas más tarde. El Convenio permite a los Estados Miembros de la OIT, cuya economía y cuyos recursos médicos sean insuficientemente desarrollados, que tomen ventaja de las excepciones temporales relativas, por ejemplo, en la extensión de la cobertura de personal en la aplicación de las ramas la seguridad social ${ }^{16}$.

El Convenio contiene, igualmente, una serie de normas comunes para todas las contingencias cubiertas ${ }^{17}$. Estas reglas definen particularmente los casos en que las prestaciones pueden ser suspendidas; también trata del derecho a un recurso de las partes y establece los principios en materia de la financiación. El Estado debe, además, asumir la responsabilidad general respecto al suministro de las prestaciones y la administración de las instituciones y servicios. Por último, los representantes de las personas protegidas deberán participar en la administración de la seguridad social o ser asociados, dándoles un poder consultivo, cuando dicha administración no esté confiada a una institución reglamentada por las autoridades públicas o por un departamento gubernamental.

social, Informe IV (2), Cuarto punto del orden del día, Conferencia Internacional del Trabajo, Trigésima cuarta reunión-1951, Ginebra, 1950.

13 Sobre este convenio ver: BIT, "Etablir des normes de sécurité sociale dans une société mondialisée. Une analyse de la situation et de la pratique présentes, ainsi que des options futures pour l'établissement pour l'Organisation internationale du travail d'une norme de sécurité sociale globale", Campagne mondiale sur la sécurité sociale et la couverture pour tous. Document de politique de sécurité sociale, Département de la sécurité sociale, Genève, mars 2008; KULKE, U., "Le rôle actuel et futur des normes de l'OIT dans la mise en œuvre du droit à la sécurité sociale", p. 133; KULKE, U., "Overview of Up-to-date ILO social Security Conventions", p. 27; SERVAIS, J-M., 2004, p. 225 et ss.; BIT, General Survey of the Reports relating to the Social Security (Mimimun Standars) Convention ( $\left.\mathrm{N}^{\circ} 102\right), 1952$, the Invalidity, Old-Age and Survivors'Benefits Convention $\left(\mathrm{N}^{\circ} 128\right)$ and Recommendation $\left(\mathrm{N}^{\circ} 131\right), 1967$, in so far as they apply to old-age benefits, International Labour Conference, $76^{\text {th }}$ session, Geneva, 1989; BIT, Rapport de la Commission d'Experts pour l'application des conventions et recommandations. Conférence Internationale du Travail, $45^{\circ}$ session, Genève, 1961; DupeyrouX, J-J., "Le droit à la sécurité sociale dans les déclarations et pactes internationaux”, p. 365; DURAND, P., 1953, réed 2005, p. 422 et ss.

14 Ver nota 6.

15 Artículo 2 del Convenio No 102

16 Artículo 3 del Convenio No 102

17 Parte XIII del Convenio No 102 
La Parte VI del Convenio No 102 está dedicada a las "Prestaciones en Caso de Accidente del Trabajo y de Enfermedad Profesional”. Las contingencias cubiertas deberán ser las siguientes ${ }^{18}$ :

a) el estado mórbido;

b) la incapacidad para trabajar que resulte de un estado mórbido y que entrañe la suspensión de ganancias, según lo defina la legislación nacional;

c) la pérdida total de la capacidad para ganar o la pérdida parcial que exceda de un grado prescrito, cuando sea probable que dicha pérdida total o parcial sea permanente, o disminución correspondiente de las facultades físicas; y

d) pérdida de medios de existencia sufrida por la viuda o los hijos como consecuencia de la muerte del sostén de familia; en el caso de la viuda, el derecho a las prestaciones puede quedar condicionado a la presunción, conforme a la legislación nacional, de que es incapaz de subvenir a sus propias necesidades.

El campo de aplicación de la cobertura de esta rama debe comprender ${ }^{19}$ :

a) a categorías prescritas de asalariados que en total constituyan, por lo menos, el 50\% de todos los asalariados, $\mathrm{y}$, para las prestaciones a que da derecho la muerte del sostén de familia, también a las cónyuges y a los hijos de los asalariados de esas categorías; o

b) cuando se haya formulado una declaración en virtud del artículo 3, a categorías prescritas de asalariados que en total constituyan, por lo menos, el $50 \%$ de todos los asalariados que trabajen en empresas industriales en las que estén empleadas, como mínimo, veinte personas, y, para las prestaciones a que da derecho la muerte del sostén de familia, también a los cónyuges y a los hijos de los asalariados de esas categorías.

Las prestaciones de esta rama comprenden tanto la asistencia médica para cubrir los estados mórbidos y pagos periódicos para reemplazar los ingresos en caso de incapacidad. En cuanto a la asistencia médica, ella tendrá por objeto conservar, reestablecer o mejorar la salud de la persona protegida, así como su aptitud para el trabajo y para hacer frente a sus necesidades personales. Esta debe comprender ${ }^{20}$ :

a) la asistencia médica general y la ofrecida por especialistas, a personas hospitalizadas o no hospitalizadas, comprendidas las visitas a domicilio;

b) la asistencia odontológica;

c) la asistencia por enfermeras, a domicilio, en un hospital o en cualquier otra institución médica;

\footnotetext{
18 Artículo 32 del Convenio $\mathrm{N}^{\circ} 102$

19 Artículo 33 del Convenio No 102

20 Según el Artículo 34 del Convenio No 102 párrafo 2. Sin embargo, cuando se haya formulado una declaración en virtud del artículo 3, la asistencia médica deberá comprender, por lo menos: a) la asistencia médica general, comprendidas las visitas a domicilio; b) la asistencia por especialistas, ofrecida en hospitales a personas hospitalizadas o no hospitalizadas, y la asistencia que pueda ser prestada por especialistas fuera de los hospitales; c) el suministro de productos farmacéuticos esenciales recetados por médicos u otros profesionales calificados; y d) la hospitalización, cuando fuere necesaria; de acuerdo al Artículo 34 del Convenio $\mathrm{N}^{\circ} 102$, párrafo 3 .
} 
d) el mantenimiento en un hospital, centro de convalecencia, sanatorio u otra institución médica;

e) el suministro de material odontológico, farmacéutico, y cualquier otro material médico o quirúrgico, comprendidos los aparatos de prótesis y su conservación, así como los anteojos; $y$

f) la asistencia suministrada por miembros de otras profesiones reconocidas legalmente como conexas con la profesión médica, bajo la vigilancia de un médico o de un dentista.

La cobertura de asistencia médica no se agota con el restablecimiento de la salud del trabajo. El convenio No 102 agrega que los departamentos gubernamentales o las instituciones que concedan la asistencia médica deberán cooperar, cuando fuere oportuno, con los servicios generales de reeducación profesional, a fin de readaptar para un trabajo apropiado a las personas de capacidad reducida. Además señala que la legislación nacional podrá autorizar a dichos departamentos o instituciones para que tomen medidas destinadas a la reeducación profesional de las personas con capacidad reducida ${ }^{21}$.

Con respecto a los pagos periódicos el Convenio No 102 señala que en los casos de incapacidad para trabajar o de pérdida total de capacidad para ganar, cuando es probable que sea permanente a la disminución correspondiente de las facultades físicas o a la muerte del sostén de familia, la prestación deberá consistir en un pago periódico ${ }^{22}$. Sin embargo, para el caso de pérdida parcial de la capacidad para ganar, cuando es probable que sea permanente, o en caso de una disminución correspondiente de las facultades físicas, la prestación, cuando deba ser pagada, consistirá en un pago periódico proporcional a la prestación prevista en caso de pérdida total de la capacidad para ganar o de una disminución correspondiente de las facultades físicas. Finalmente agrega que los pagos periódicos podrán sustituirse por un capital pagado de una sola vez cuando la legislación considere una de las siguientes situaciones:

a) el grado de incapacidad sea mínimo; o

b) se garantice a las autoridades competentes el empleo razonable de dicho capital.

En cuanto a los pagos periódicos, el Convenio No 102 ha establecido el siguiente cuadro para determinar el cálculo de la prestación en función de los beneficiarios tipo, distinguiendo de cual contingencia se trate. Para el caso de la parte VI son las siguientes:

\begin{tabular}{|l|l|l|l|}
\hline \multicolumn{1}{|c|}{ Partes } & \multicolumn{1}{c|}{ Contingencias } & \multicolumn{1}{c|}{ Beneficiarios tipo } & Porcentaje \\
\hline Accidentes del trabajo y enfermedades profesionales: & 50 \\
\hline VI & Incapacidad para trabajar & Hombre con cónyuge y dos hijos & 50 \\
\hline VI & Invalidez & Hombre con cónyuge y dos hijos & 40 \\
\hline VI & Sobrevivientes & Viuda con dos hijos & 40 \\
\hline
\end{tabular}

\footnotetext{
21 Artículo 35 del Convenio No 102
}

22 Artículo 36 del Convenio No 102 
El convenio No 102 destaca que las prestaciones de asistencia medica y de pagos periódicos deberán garantizarse, para la contingencia cubierta, por lo menos a las personas protegidas que estuvieran empleadas como asalariados en el territorio del Estado miembro al momento del accidente o en el que se contrajo la enfermedad; y si se trata de pagos periódicos resultantes del fallecimiento del sostén de familia, a la viuda y a los hijos de aquel ${ }^{23}$. Además, las prestaciones deben concederse durante toda la duración de la contingencia, sin embargo, con respecto a la incapacidad para trabajar, la prestación podrá no pagarse por los tres primeros días en cada caso de suspensión de ganancias, estableciendo así la posibilidad de fijar un período de carencia de máximo tres días ${ }^{24}$.

\section{EL CONVENIO 121 SOBRE LAS PRESTACIONES EN CASO DE ACCIDENTES Y ENFERMEDADES PROFESIONALES}

En su 150a Reunión, celebrada en noviembre de 1961, el Consejo de Administración de la OIT decidió incluir en el orden del día de la 47a Reunión de la Conferencia Internacional del Trabajo, de 1963, el tema: "Prestaciones en caso de accidentes de trabajo y enfermedades profesionales". Para la preparación de la primera discusión, la Oficina preparó su Informe VII (1), "Prestaciones en caso de accidentes de trabajo y enfermedades profesionales", de 1962. Posteriormente la Conferencia Internacional del Trabajo, después de haber decidido adoptar diversas proposiciones relativas a las prestaciones en caso de accidentes del trabajo y enfermedades profesionales, optó que dichas proposiciones revistieran la forma de un convenio internacional, por lo que adoptó, con fecha 8 de julio de 1964, el Convenio No 121 sobre las prestaciones en caso de accidentes del trabajo y enfermedades profesionales.

Como su denominación lo indica, el Convenio se dirige especialmente a considerar las prestaciones que se deben brindar ante los accidentes del trabajo y las enfermedades profesionales. Tal como lo estipula el Convenio No 102, el Convenio No 121 permite, en su artículo 2, a los Estados Miembros de la OIT, cuya economía y cuyos recursos médicos sean insuficientemente desarrollados, que tomen ventaja de las excepciones temporales relativas, lo que reafirma el carácter flexible de las normas internacionales de la OIT. Además, el Convenio No 121 permite excluir de su campo de aplicación, mediante una declaración anexa, a la gente de mar, incluidos los pescadores de pesquerías marítimas y a los funcionarios públicos, en este último caso cuando estas categorías estén protegidas en virtud de regímenes especiales que concedan en conjunto prestaciones por lo menos equivalentes a las del presente Convenio ${ }^{25}$.

El Convenio prescribe que la legislación nacional sobre las prestaciones en caso de accidentes del trabajo y enfermedades profesionales debe proteger a todos los asalariados, incluidos los aprendices, de los sectores público y privado, comprendidos aquellos de las cooperativas, y, en caso de fallecimiento del sostén de familia, a categorías prescritas de beneficiarios. Permite que se excluya del campo de aplicación personal a las personas que realicen

\footnotetext{
23 Artículo 37 del Convenio No 102

24 Artículo 38 del Convenio No 102

25 Artículo 3 del Convenio No 121
} 
trabajos ocasionales ajenos a la empresa del empleador; a los trabajadores a domicilio; a los miembros de la familia del empleador que vivan con él y respecto del trabajo que realicen para él; a otras categorías de asalariados dentro de ciertos márgenes establecidos ${ }^{26}$.

Las contingencias cubiertas en materia de accidentes de trabajo y enfermedades profesionales son: el estado mórbido, la incapacidad para trabajar que resulte de un estado mórbido y que entrañe suspensión de ganancias, la pérdida total o parcial para ganar, y la pérdida de medios de existencia, sufrida a consecuencia del fallecimiento del sostén de familia ${ }^{27}$.

Los Estados miembros que ratifican este Convenio deben efectuar una definición de "accidente de trabajo" y deben establecer las condiciones bajo las cuales debe considerarse como tal un accidente sufrido en el trayecto del hogar al lugar de trabajo ${ }^{28}$. Además, deben definir el concepto de enfermedad profesional y prescribir una lista de ellas. Para esto, el Convenio presenta un "Cuadro de enfermedades profesionales" 29.

En cuanto a las prestaciones, cada Estado miembro deberá garantizar a las personas protegidas dos tipos de prestaciones ${ }^{30}$ :

a) asistencia médica y servicios conexos en caso de estado mórbido;

b) prestaciones monetarias.

El inicio del derecho a la prestación no puede ser subordinado ni a la duración del tiempo del empleo ni a la duración del período de afiliación al seguro o al pago de las cotizaciones. Sin embargo, en lo relativo a las enfermedades profesionales puede establecerse un período de exposición al riesgo previsto. Las prestaciones se otorgaran mientras exista la situación que les da derecho a ellas. Sin embargo, en cuanto a la incapacidad para el trabajo, la prestación monetaria podrá no ser pagadera durante los tres primeros días, en los siguientes $\operatorname{casos}^{31}$ :

a) cuando la legislación de un Estado miembro, en la fecha en que este Convenio entre en vigor, establezca un período de espera y así lo haga saber a la organización.

b cuando esté en vigor una declaración formulada de conformidad con lo previsto en el artículo 2 .

Las prestaciones de asistencia médica así como los servicios conexos en caso de estado mórbido son bastante más generosas que el Convenio No 102 . Se exige que deba dispensar-

\footnotetext{
26 Artículo 4 del Convenio No 121

27 Artículo 6 del Convenio No 121

28 Artículo 7 párrafo 1 del Convenio No 121. Sin embargo, el párrafo $2^{\circ}$ del mismo artículo señala: 2. No será necesario incluir en la definición de accidentes del trabajo las condiciones bajo las cuales debe considerarse como tal un accidente sufrido en el trayecto si, independientemente de los sistemas de seguridad social que cubren los accidentes del trabajo y enfermedades profesionales, hay otros sistemas distintos que cubren tales accidentes sufridos en el trayecto, y que conceden prestaciones que en su conjunto son por lo menos equivalentes a las que establece este Convenio.

29 Artículo 8 del Convenio No 121

30 Artículo 9 párrafo 1 Convenio No 121

31 Artículo 9 párrafos 2 y 3 Convenio No 121
} 
se por todos los medios apropiados, a fin de conservar, restablecer o, si esto no fuera posible, mejorar la salud de la víctima, así como su aptitud para trabajar y para hacer frente a sus necesidades personales. Además de recuperar la salud se debe recuperar la aptitud para trabajar. Se deberá comprender las prestaciones establecidas en el artículo 10 del Convenio, a saber:

a) la asistencia médica general y la ofrecida por especialistas a personas hospitalizadas o no hospitalizadas, incluidas las visitas a domicilio;

b) la asistencia odontológica;

c) la asistencia por enfermeras, a domicilio, en un hospital o en cualquier otra institución médica;

d) el mantenimiento en un hospital, centro de convalecencia, sanatorio u otra institución médica;

e) el suministro del material odontológico, farmacéutico y cualquier otro material médico o quirúrgico, comprendidos los aparatos de prótesis y su conservación, reparación y renovación cuando sea necesario, así como los lentes;

f) la asistencia suministrada, bajo la vigilancia de un médico o de un dentista, de tratamientos por miembros de otras profesiones reconocidas legalmente como conexas con la profesión médica; $y$

g) en la medida de lo posible, el siguiente tratamiento en el lugar de trabajo:

i) tratamiento de urgencia a las víctimas de accidentes graves;

ii) cuidados ulteriores a las víctimas de lesiones leves que no acarreen interrupción del trabajo.

En cuanto a las prestaciones monetarias por incapacidad temporal o inicial para el trabajo, se harán en forma de pago periódico. Los artículos 19 y 20 establecen la forma como deben efectuarse el cálculo de las prestaciones monetarias ${ }^{32}$ que sigue la línea del método establecido en el Convenio No 102 y se consagra igualmente un cuadro con los beneficiarios tipo y las tasas de remplazo.

\begin{tabular}{|l|l|l|}
\hline \multicolumn{1}{|c|}{ Contingencias } & \multicolumn{1}{|c|}{ Beneficiario tipo } & \multicolumn{1}{c|}{ Porcentaje } \\
\hline 1. Incapacidad temporal o inicial para trabajar. & Hombre con cónyuge y dos hijos. & 60 \\
\hline $\begin{array}{l}\text { 2. Pérdida total de la capacidad para ganar o disminución } \\
\text { correspondiente de las facultades físicas. }\end{array}$ & Hombre con cónyuge. & 60 \\
\hline 3. Fallecimiento del sostén de la familia. & Viuda con dos hijos. & 50 \\
\hline
\end{tabular}

Se estipula una prestación por pérdida de capacidad por ganar o por disminución de las facultades físicas y se indica que, en caso de pérdida total de la capacidad para ganar, cuando sea probable que esta sea permanente, o en caso de disminución correspondiente de las facultades físicas, la prestación monetaria consistirá en un pago periódico calculado de conformidad con las disposiciones del artículo 19 o con las del artículo 20. En caso de pérdida parcial sustancial de la capacidad para ganar que exceda un porcentaje prescrito, y

32 Artículo 13 del Convenio $\mathrm{N}^{\circ} 121$ 
cuando sea probable que esta pérdida sea permanente, o en caso de disminución correspondiente de las facultades físicas, la prestación consistirá en un pago periódico que representará una proporción conveniente de la prestación prevista en el caso anterior. En caso de cualquier otra pérdida parcial de la capacidad de ganar que exceda del porcentaje prescrito y cuando sea probable que esta pérdida sea permanente, o en caso de disminución correspondiente de las facultades físicas, la prestación monetaria podrá adoptar la forma de una suma global (llámese también capital) ${ }^{33}$.

Siguiendo el esquema impuesto en el Convenio No 102, se establecen normas relativas a principios generales de la seguridad social. Así, se reglamenta la suspensión de la prestación ${ }^{34}$, la apelación ${ }^{35}$, la administración y representación de los asegurados ${ }^{36}$, la responsabilidad general del Estado ${ }^{37}$ y la igualdad de trato ${ }^{38}$. Pero además, el artículo 26 del Convenio, incorpora las obligaciones de tomar medidas de prevención contra los accidentes del trabajo y las enfermedades profesionales; proporcionar servicios de readaptación profesional que, cuando sea posible, preparen a la persona incapacitada para reanudar sus actividades anteriores o, si esto no pudiera realizarse, para ejercer la actividad lucrativa más adecuada, en la medida posible, a su actividad anterior, habida cuenta de sus calificaciones y aptitudes; y tomar medidas para facilitar la colocación adecuada de los trabajadores que hayan quedado inválidos.

Se establece la posibilidad, en "circunstancias excepcionales", con el consentimiento de la víctima, y cuando la autoridad competente tenga motivos para creer que el pago de una suma global puede ser muy ventajosa para el beneficiario, de cambiar el total o parte de los pagos periódicos por un capital correspondiente al equivalente actuarial de los pagos periódicos ${ }^{39}$. Y, cuando las personas incapacitadas necesitan de asistencia constante de otra persona, se pueden establecer incrementos de los pagos periódicos ${ }^{40}$.

Se establecen prestaciones monetarias en caso de fallecimiento del sostén de la familia que consistirán en un pago periódico a las siguientes personas: a la viuda, de acuerdo con lo que prescriba la legislación nacional; al viudo, si se encuentra a cargo del incapacitado; a los hijos a cargo del fallecido, y a toda otra persona que fuera designada por la legislación nacional. El pago debe ser calculado de acuerdo a lo establecido en los artículos 19 y 20. Se establece además una prestación para gastos funerarios a una tasa prescrita. El derecho a esta prestación podrá ser subordinado a las condiciones prescritas cuando las prestaciones monetarias a los sobrevivientes sean notablemente superiores a las que establece el presente Convenio ${ }^{41}$.

\footnotetext{
3 Artículo 14 del Convenio No 121

34 Artículo 22 del Convenio No 121

35 Artículo 23 del Convenio No 121

36 Artículo 24 del Convenio No 121

37 Artículo 25 del Convenio No 121

38 Artículo 27 del Convenio No 121

39 Artículo 15 del Convenio No 121

40 Artículo 16 del Convenio No 121

41 Artículo 18 Convenio No 121
} 
En su artículo 28 el Convenio señala que revisa los Convenios No 12 sobre la indemnización por accidentes del trabajo (agricultura), de 1921; No 17 sobre la indemnización por accidentes del trabajo, de 1925; No 18 sobre las enfermedades profesionales, de 1925; y N 42 sobre las enfermedades profesionales (revisado), de 1934. La ratificación del Convenio No 121 por un Estado que hubiese ratificado anteriormente el Convenio No 42 sobre las enfermedades profesionales (revisado), de 1934, implicará, ipso jure, la denuncia inmediata de dicho Convenio, de conformidad con su artículo 8, al entrar en vigor el presente Convenio, pero la entrada en vigor del presente Convenio no cerrará a la ratificación el Convenio sobre las enfermedades profesionales (revisado), de 1934. Además, se especifica en su artículo 29 que de conformidad con el artículo 75 del Convenio No 102 sobre la seguridad social (norma mínima), de 1952, la parte VI y las disposiciones correspondientes de otras partes de dicho Convenio cesarán de aplicarse a todo Estado que ratifique el Convenio No 121, a partir de la fecha de su entrada en vigor para ese miembro. No obstante, se considerará que la aceptación de las obligaciones del presente Convenio constituye una aceptación de las obligaciones de la parte VI y de las disposiciones pertinentes de otras partes del Convenio No 102 sobre la seguridad social (norma mínima), de 1952.

Señalemos que la Conferencia Internacional del Trabajo, en 1980, adoptó una enmienda de la lista de enfermedades profesionales anexa al Convenio No 121 sobre las prestaciones en caso de accidentes del trabajo y enfermedades profesionales, de 1964, que fue sometida a la Conferencia por la Comisión de Seguridad e Higiene ${ }^{42}$.

Por otro lado, debemos mencionar que el Convenio No 121 fue completado por la Recomendación $\mathrm{n}^{\circ} 121$ que apunta a la extensión de las prestaciones de accidentes del trabajo y de enfermedades profesionales a categorías de trabajadores que habían sido excluidas (por ejemplo, estudiantes), así como también a los miembros de las cooperativas, y a ciertas categorías de trabajadores independientes.

El Convenio No 121 sobre las prestaciones en caso de accidentes del trabajo y enfermedades profesionales de 1964, fue ratificado por Chile el 30 de septiembre de 1999, siendo así el único convenio de tercera generación en materia de seguridad social que ha ratificado nuestro país. Destaquemos, además, que este Convenio ha sido declarado como al día, y por tanto su contenido está plenamente vigente a las necesidades actuales según la OIT.

A continuación, dentro de un afán metodológico y sistemático hemos decidido incluir un cuadro resumen de la protección de los accidentes del trabajo y enfermedades profesionales que otorgan los convenios No 102 y No 121 .

42 Ver: OIT, Actas de las sesiones. Conferencia Internacional del Trabajo, 66a reunión-1980. Ginebra, 1981. 


\section{CuAdro 1}

PRESTACIONES EN CASO DE ACCIDENTE DEL TRABAJO Y DE ENFERMEDAD PROFESIONAL

\begin{tabular}{|c|c|c|}
\hline & Convenio No 102 & Convenio No 121 \\
\hline $\begin{array}{l}\text { Campo de } \\
\text { aplicación }\end{array}$ & $\begin{array}{c}50 \% \text { de asalariados. } \\
\text { Declaración artículo } 3 .\end{array}$ & Todos los asalariados. \\
\hline $\begin{array}{l}\text { Naturaleza de las } \\
\text { prestaciones }\end{array}$ & $\begin{array}{l}\text { - Salvo en los casos de incapacidad } \\
\text { de trabajo, obligación de revisar } \\
\text { estos montos en caso de variaciones } \\
\text { sensibles del costo de la vida. } \\
\text { - Posibilidad de convertir los pagos } \\
\text { periódicos en un capital únicamente } \\
\text { 1) cuando el grado de incapacidad es } \\
\text { mínimo o 2) cuando se proporciona } \\
\text { a las autoridades competentes la } \\
\text { garantía de que se hará un uso juicioso } \\
\text { de ese capital. }\end{array}$ & $\begin{array}{l}\text { - Ibid. Además, ciertos tipos de asistencia en } \\
\text { el lugar de trabajo. } \\
\text { - Pagos periódicos de, por lo menos, el } 60 \% \\
\text { del salario de referencia en los casos de } \\
\text { incapacidad temporal o de invalidez. } \\
\text { - En caso de fallecimiento del sostén de } \\
\text { familia, prestaciones para la viuda, el } \\
\text { viudo inválido y a cargo, los hijos a cargo } \\
\text { y todas las otras personas designadas por } \\
\text { la legislación nacional. Pagos periódicos } \\
\text { de, por lo menos, el 50\% del salario de } \\
\text { referencia. En principio, pago de una } \\
\text { prestación para los gastos funerarios. } \\
\text { - Obligación de fijar un monto mínimo para } \\
\text { los pagos periódicos. } \\
\text { - Ibid. } \\
\text { - Prestaciones suplementarias para las } \\
\text { víctimas cuyo estado requiere la asistencia } \\
\text { constante de una tercera persona. } \\
\text { periódicos en un capital 1) en caso de } \\
\text { pérdida no substancial de la capacidad } \\
\text { de ganar o 2) en casos excepcionales y } \\
\text { previo acuerdo de la víctima, cuando la } \\
\text { autoridad competente tiene razones para } \\
\text { creer que ese capital será utilizado de forma } \\
\text { especialmente ventajosa para la víctima. }\end{array}$ \\
\hline $\begin{array}{l}\text { Condiciones de } \\
\text { apertura del derecho }\end{array}$ & $\begin{array}{l}\text { - Prohibición de imponer un período de } \\
\text { calificación. } \\
\text { - Posibilidad de subordinar el derecho } \\
\text { a las prestaciones de la viuda a la } \\
\text { presunción de que es incapaz de } \\
\text { subvenir a sus propias necesidades. }\end{array}$ & $\begin{array}{l}\text { - Ibid. } \\
\text { - Posibilidad para las autoridades nacionales } \\
\text { de prescribir las condiciones en las } \\
\text { cuales una viuda puede pretender recibir } \\
\text { prestaciones. }\end{array}$ \\
\hline $\begin{array}{l}\text { Duración de las } \\
\text { prestaciones }\end{array}$ & $\begin{array}{l}\text { - No hay plazo de carencia, excepto en } \\
\text { los casos de incapacidad temporal de } \\
\text { trabajo (3 días como máximo). }\end{array}$ & $\begin{array}{l}\text { - Posibilidad de fijar un plazo de carencia } \\
\text { para los casos de incapacidad de trabajo, si } \\
\text { este plazo estaba previsto en la legislación } \\
\text { en el momento de entrada en vigor del } \\
\text { Convenio y si las razones para prevalerse } \\
\text { de él siguen existiendo. } \\
\text { - Ibid. }\end{array}$ \\
\hline
\end{tabular}

Fuente: Humblet, M.; SILVA, R. Normas para el siglo XXI, Seguridad Social. Ginebra, OIT, 2002. 


\section{PALABRAS FINALES}

Nuestro país ha ratificado normas internacionales del trabajo de la primera y de la tercera generación. De la primera generación ha ratificado los convenios sobre esta materia, pero posteriormente los ha denunciado, lo que significa que actualmente nuestro país no se encuentra obligado por estas normas.

Por otro lado, de lo expuesto nos llama la atención que Chile no haya ratificado el Convenio $\mathrm{N}^{\circ} 102$, ya que para su ratificación basta con que el país ratifique 3 de sus ramas. El artículo 29 del convenio No 121 señala "que se considerará que la aceptación de las obligaciones del presente Convenio constituye una aceptación de las obligaciones de la parte VI y de las disposiciones pertinentes de otras partes del Convenio sobre la seguridad social (norma minima), 1952”. Dicho de una manera más simple, el hecho que un país haya ratificado un Convenio de tercera generación implica la aceptación al mismo tiempo de la parte correspondiente del Convenio No 102. O sea, nuestro país ha aceptado la parte VI del Convenio $\mathrm{N}^{\circ} 102$, faltándole, tan solo dos partes más. A nuestro parecer Chile cumple con las condiciones de ratificación de este convenio y pensamos que su no ratificación ha pasado por un desconocimiento de su contenido y por una falta de voluntad política al respecto.

Por otro lado, dentro de las normas de la tercera generación, como ya lo hemos señalado, Chile ha ratificado el Convenio $N^{\circ} 121$ en 1999 y se encuentra obligado por sus disposiciones. Pero, sin embargo, ha sido objeto de observaciones y de una solicitud directa por parte de la Comisión de Expertos en Aplicación de Convenios y Recomendaciones de la $\mathrm{OIT}^{43}$

Por último, debemos señalar que la ratificación de los Convenios No 102 y No 121 es bastante dispar. El Convenio No 102 posee 47 ratificaciones y el Convenio No 121 solo 24 . Como lo vemos en los anexos existen países que han ratificados ambos instrumentos y otros que tan solo han ratificado uno de ellos. Nuestro país es de aquellos que tan solo ha ratificado el Convenio No 121.

A nuestro parecer el Estado chileno podría reforzar su compromiso en la protección de los accidentes del trabajo y de las enfermedades profesionales adoptando dos tipos de medidas. Por un lado, ratificando el Convenio No 102, otorgándole así una solidez al sistema de seguridad social chileno. Por otro, ajustando más estrictamente su legislación al Convenio No 121. Recordemos que la protección de los accidentes del trabajo y de las enfermedades profesiones, y en general de los riesgos profesionales, constituye la base de todo sistema de seguridad social.

\footnotetext{
43 Las observaciones de la CEACR son: CEACR: Observación individual sobre el Convenio No 121. Prestaciones en caso de accidentes del trabajo y enfermedades profesionales, 1964, Chile (ratificación: 1999.) Publicación: 2005; CEACR: Observación individual sobre el Convenio sobre las prestaciones en caso de accidentes del trabajo y enfermedades profesionales, 1964 (Cuadro I modificado en 1980) (No 121) Chile (ratificación: 1999). Publicación: 2007; y CEACR: Observación individual sobre el Convenio sobre las prestaciones en caso de accidentes del trabajo y enfermedades profesionales, 1964 [Cuadro I modificado en 1980] (No 121) Chile (ratificación: 1999). Publicación: 2008. Además la CEACR ha emitido una solicitud directa al gobierno chileno: CEACR: Solicitud directa individual sobre el Convenio No 121, prestaciones en caso de accidentes del trabajo y enfermedades profesionales, 1964, Chile (ratificación: 1999). Envío: 2005. Los textos de las observaciones y de la solicitud directa pueden ser consultados en el sitio www.ilo.org.
} 


\section{BIBLIOGRAFÍA}

AREllano OrTIZ, Pablo. "Reflexiones sobre las evoluciones recientes en materia de protección de riesgos sociales a nivel internacional”, en: Revista de Derecho Universidad Católica del Norte, vol. 17, № 2, 2010, pp. 167-183.

Humblet, M.; Silva, R. Normas para el siglo XXI, Seguridad Social. Ginebra, OIT, 2002.

Lyon-Caen, G.; LyOn-Caen, A. Droit social international et européen. 7éme Edition. Dalloz, París, 1991.

OIT. Establecimiento de normas de seguridad social en una sociedad global. Análisis de la situación y de la práctica actuales $y$ de las opciones futuras para el establecimiento de normas de seguridad social globales en la organización Internacional del Trabajo. Campaña Mundial sobre Seguridad Social y Cobertura para Todos, Documentos de política de seguridad social No 2, Departamento de Seguridad Social. Ginebra, 2008.

OIT. Seguridad social: un nuevo consenso. Ginebra, 2002.

OIT. Las normas internacionales del trabajo, Un enfoque global. Ginebra, 2002

OIT. Trabajo Decente. Memoria del Director General, Conferencia Internacional del Trabajo, 87a reunión. Ginebra, 1999.

OIT. Actas de las sesiones. Conferencia Internacional del Trabajo, 66a reunión-1980. Ginebra, 1981.

OIT. Acta de las sesiones. Conferencia Internacional del Trabajo. Trigésima quinta reunión-1952. Ginebra, 1953.

OIT. Norma minima de la seguridad social, Informe V a) (1). Quinto punto del orden del día. Conferencia Internacional del Trabajo. Trigésima quinta reunión-1952. Ginebra, 1951

OIT. Norma mínima de la seguridad social, Informe V a) (2). Quinto punto del orden del día, Conferencia Internacional del Trabajo. Trigésima quinta reunión-1952. Ginebra, 1952.

OIT. Objetivos y norma avanzada de la seguridad social. Informe V b). Quinto punto del orden del día. Conferencia Internacional del Trabajo. Trigésima quinta reunión-1952. Ginebra, 1952

OIT. Objetivos y normas mínimas de la seguridad social. Informe IV (1). Cuarto punto del orden del día. Conferencia Internacional del Trabajo. Trigésima cuarta reunión-1951. Ginebra, 1950

OIT. La seguridad social: estudio internacional. Estudios y documentos. Nueva serie No 23. Ginebra, 1950.

OIT. Objetivos y normas minimas de la seguridad social. Informe IV (2), Cuarto punto del orden del día. Conferencia Internacional del Trabajo. Trigésima cuarta reunión-1951. Ginebra, 1950.

RODRÍGUEZ, C. A. Los convenios de la OIT sobre seguridad y salud en el trabajo: una oportunidad para mejorar las condiciones y el medio ambiente de trabajo. Buenos Aires: Oficina de la OIT Argentina, Centro Internacional de Formación de la OIT, Turín-CIF, 2009.

SERVAIS, J.-M. Normes Internationales du Travail, L.G.D.J. París, 2004.

VAlTiCos, N. Droit International du Travail. 2e édition. Dalloz, 1983. 


\section{ANEXO 1. INSTRUMENTOS DE LA OIT SOBRE PRESTACIONES EN CASO DE ACCIDENTES DEL TRABAJO Y ENFERMEDADES PROFESIONALES}

\section{INSTRUMENTOS ACTUALIZADOS}

a) Convenio (No 121) sobre las prestaciones en caso de accidentes del trabajo y enfermedades profesionales, de 1964.

b) Recomendación ( $\mathrm{N}^{\circ}$ 121) sobre las prestaciones en caso de accidentes del trabajo y enfermedades profesionales, de 1964.

\section{INSTRUMENTOS EN SITUACIÓN PROVISORIA}

a) Convenio (No 12) sobre la indemnización por accidentes del trabajo (agricultura), de 1921.

\section{INSTRUMENTOS QUE HAN SIDO SUPERADOS}

a) Convenio (No 17) sobre la indemnización por accidentes del trabajo, de 1925.

b) Convenio (No 18) sobre las enfermedades profesionales, de 1925.

Recomendación (№ 24) sobre las enfermedades profesionales, de 1925.

Convenio (No 42) sobre las enfermedades profesionales (revisado), de 1934.

Recomendación (No 22) sobre la indemnización por accidentes del trabajo (importe mínimo), de 1925.

Recomendación (No 23) sobre la indemnización por accidentes del trabajo (jurisdicción), de 1925.

ANEXO 2. CUADRO ESTADO DE RATIFICACIÓN DEL CONVENIO Nº 102: TOTAL PAÍSES 47

\begin{tabular}{|l|c|}
\hline \multicolumn{1}{|c|}{ PAÍS } & $\begin{array}{c}\text { Fecha de } \\
\text { ratificación }\end{array}$ \\
\hline Albania & $18 / 01 / 2006$ \\
\hline Alemania & $21 / 02 / 1958$ \\
\hline Austria & $04 / 11 / 1969$ \\
\hline Barbados & $11 / 07 / 1972$ \\
\hline Bélgica & $26 / 11 / 1959$ \\
\hline $\begin{array}{l}\text { Estado Plurinacional } \\
\text { de Bolivia }\end{array}$ & $31 / 01 / 1977$ \\
\hline $\begin{array}{l}\text { Bosnia y } \\
\text { Herzegovina }\end{array}$ & $02 / 06 / 1993$ \\
\hline Brasil & $15 / 06 / 2009$ \\
\hline Bulgaria & $14 / 07 / 2008$ \\
\hline Chipre & $03 / 09 / 1991$ \\
\hline $\begin{array}{l}\text { República } \\
\text { Democrática del } \\
\text { Congo }\end{array}$ & $03 / 04 / 1987$ \\
\hline Costa Rica & $16 / 03 / 1972$ \\
\hline Croacia & $08 / 10 / 1991$ \\
\hline Dinamarca & $15 / 08 / 1955$ \\
\hline
\end{tabular}

\begin{tabular}{|l|l|}
\hline \multicolumn{1}{|c|}{ PAÍS } & $\begin{array}{c}\text { Fecha de } \\
\text { ratificación }\end{array}$ \\
\hline Ecuador & $25 / 10 / 1974$ \\
\hline Eslovaquia & $01 / 01 / 1993$ \\
\hline Eslovenia & $29 / 05 / 1992$ \\
\hline España & $29 / 06 / 1988$ \\
\hline Francia & $14 / 06 / 1974$ \\
\hline Grecia & $16 / 06 / 1955$ \\
\hline Irlanda & $17 / 06 / 1968$ \\
\hline Islandia & $20 / 02 / 1961$ \\
\hline Israel & $16 / 12 / 1955$ \\
\hline Italia & $08 / 06 / 1956$ \\
\hline $\begin{array}{l}\text { Jamahiriya Arabe } \\
\text { Libia }\end{array}$ & $19 / 06 / 1975$ \\
\hline Japón & $02 / 02 / 1976$ \\
\hline Luxemburgo & $31 / 08 / 1964$ \\
\hline $\begin{array}{l}\text { Ex República } \\
\text { Yugoslava de } \\
\text { Macedonia }\end{array}$ & $17 / 11 / 1991$ \\
\hline Mauritania & $15 / 07 / 1968$ \\
\hline
\end{tabular}

\begin{tabular}{|l|c|}
\hline \multicolumn{1}{|c|}{ PAÍS } & $\begin{array}{c}\text { Fecha de } \\
\text { ratificación }\end{array}$ \\
\hline México & $12 / 10 / 1961$ \\
\hline Montenegro & $03 / 06 / 2006$ \\
\hline Níger & $09 / 08 / 1966$ \\
\hline Noruega & $30 / 09 / 1954$ \\
\hline Países Bajos & $11 / 10 / 1962$ \\
\hline Perú & $23 / 08 / 1961$ \\
\hline Polonia & $03 / 12 / 2003$ \\
\hline Portugal & $17 / 03 / 1994$ \\
\hline Reino Unido & $27 / 04 / 1954$ \\
\hline República Checa & $01 / 01 / 1993$ \\
\hline Rumania & $15 / 10 / 2009$ \\
\hline Senegal & $22 / 10 / 1962$ \\
\hline Serbia & $24 / 11 / 2000$ \\
\hline Suecia & $12 / 08 / 1953$ \\
\hline Suiza & $18 / 10 / 1977$ \\
\hline Turquía & $29 / 01 / 1975$ \\
\hline Uruguay & $14 / 10 / 2010$ \\
\hline $\begin{array}{l}\text { Rep. Bolivariana de } \\
\text { Venezuela }\end{array}$ & $05 / 11 / 1982$ \\
\hline & \\
\hline
\end{tabular}

Fuente: ILOLEX (visitado el 8 de agosto de 2011). 
ANEXO 3. CUADRO ESTADO DE RATIFICACIÓN DEL CONVENIO N 121: TOTAL PAÍSES 24.

\begin{tabular}{|l|c|}
\hline \multicolumn{1}{|c|}{ PAIS } & $\begin{array}{c}\text { Fecha de } \\
\text { ratificación }\end{array}$ \\
\hline Alemania & $01 / 03 / 1972$ \\
\hline Bélgica & $22 / 04 / 1970$ \\
\hline $\begin{array}{l}\text { Estado Plurinacional } \\
\text { de Bolivia }\end{array}$ & $31 / 01 / 1977$ \\
\hline $\begin{array}{l}\text { Bosnia y } \\
\text { Herzegovina }\end{array}$ & $02 / 06 / 1993$ \\
\hline Chile & $30 / 09 / 1999$ \\
\hline Chipre & $28 / 07 / 1966$ \\
\hline $\begin{array}{l}\text { Rep. Democrática } \\
\text { del Congo }\end{array}$ & $05 / 09 / 1967$ \\
\hline
\end{tabular}

\begin{tabular}{|l|c|}
\hline \multicolumn{1}{|c|}{ PAIS } & $\begin{array}{c}\text { Fecha de } \\
\text { ratificación }\end{array}$ \\
\hline Croacia & $08 / 10 / 1991$ \\
\hline Ecuador & $05 / 04 / 1978$ \\
\hline Eslovenia & $29 / 05 / 1992$ \\
\hline Finlandia & $23 / 09 / 1968$ \\
\hline Guinea & $11 / 08 / 1967$ \\
\hline Irlanda & $09 / 06 / 1969$ \\
\hline $\begin{array}{l}\text { Jamahiriya Arabe } \\
\text { Libia }\end{array}$ & $19 / 06 / 1975$ \\
\hline Japón & $07 / 06 / 1974$ \\
\hline Luxemburgo & $24 / 07 / 1972$ \\
\hline
\end{tabular}

\begin{tabular}{|l|c|}
\hline \multicolumn{1}{|c|}{ PAIS } & $\begin{array}{c}\text { Fecha de } \\
\text { ratificación }\end{array}$ \\
\hline $\begin{array}{l}\text { Ex Rep. Yugoslava } \\
\text { de Macedonia }\end{array}$ & $17 / 11 / 1991$ \\
\hline Montenegro & $03 / 06 / 2006$ \\
\hline Países Bajos & $02 / 08 / 1966$ \\
\hline Senegal & $25 / 04 / 1966$ \\
\hline Serbia & $24 / 11 / 2000$ \\
\hline Suecia & $17 / 06 / 1969$ \\
\hline Uruguay & $28 / 06 / 1973$ \\
\hline $\begin{array}{l}\text { Rep. Bolivariana de } \\
\text { Venezuela }\end{array}$ & $10 / 08 / 1982$ \\
\hline
\end{tabular}

Fuente: ILOLEX (visitado el 8 de agosto de 2011).

\section{ANEXO 4. CUADRO COMPARADO POR REGIÓN DE RATIFICACIONES CONVENIOS No 102 Y No 121.}

\begin{tabular}{|l|l|}
\hline $\begin{array}{c}\text { REGIÓN } \\
\text { Y PAÍS }\end{array}$ & $\begin{array}{c}\text { Accidentes del } \\
\text { Trabajo } \\
\text { C. No } 121 \text { y/o Parte } \\
\text { VI del C. No 102 }\end{array}$ \\
\hline África & \\
\hline $\begin{array}{l}\text { República } \\
\text { Democrática } \\
\text { del Congo }\end{array}$ & C. $121(1967)$ \\
\hline Guinea & C. $121(1967)$ \\
\hline $\begin{array}{l}\text { Jamahiriya } \\
\text { Árabe Libia }\end{array}$ & C. $102(1975)$ \\
\hline C. $121(1975)$ \\
\hline Niguritania & C. $102(1968)$ \\
\hline Senegal & C. $102(1966)$ \\
\hline América & C. $102(1962)$ \\
\hline Barbados & C. $121(1966)$ \\
\hline Bolivia & C. $102(1972)$ \\
\hline Brasil & C. $102(1977)$ \\
\hline Chile & C. $121(1977)$ \\
\hline Costa Rica & C. $102(2009)$ \\
\hline Ecuador & C. $121(1999)$ \\
\hline Céxico $102(1972)$ \\
\hline Uruguay & C. $102(1974)$ \\
\hline C. $121(1978)$ \\
\hline C. $102(1961)$ \\
\hline C. $102(2009)$ \\
C. $121(1973)$ \\
\hline Buente:
\end{tabular}

\begin{tabular}{|l|l|}
\hline \multicolumn{1}{|c|}{$\begin{array}{c}\text { REGIÓN } \\
\text { Y PAÍS }\end{array}$} & $\begin{array}{c}\text { Accidentes del } \\
\text { Trabajo } \\
\text { C. No } 121 \text { y/o Parte } \\
\text { VI del C. No } 102\end{array}$ \\
\hline $\begin{array}{l}\text { República } \\
\text { Bolivariana de } \\
\text { Venezuela }\end{array}$ & $\begin{array}{l}\text { C. } 102(1982) \\
\text { C. } 121 \text { (1982) }\end{array}$ \\
\hline $\begin{array}{l}\text { Medio } \\
\text { Oriente }\end{array}$ & C. $102(1955)$ \\
\hline Israel & C. $102(1976)$ \\
\hline Asia & C. $121(1974)$ \\
\hline Japón & C. $102(2006)$ \\
\hline Europa & C. $102(1959)$ \\
\hline Albania & C. $121(1970)$ \\
\hline Bélgica & C. $102(2008)$ \\
\hline Bulgaria & C. $102(1993)$ \\
\hline $\begin{array}{l}\text { Bosnia and } \\
\text { Herzegovina }\end{array}$ & C. $121(1993)$ \\
\hline Croacia & C. $102(1991)$ \\
\hline Chipre & C. $121(1991)$ \\
\hline C. $102(1991)$ \\
\hline Francia & C. $121(1966)$ \\
\hline Finlandia & C. $102(1960)$ \\
\hline & C. $102(1974)$ \\
\hline
\end{tabular}

\begin{tabular}{|l|l|}
\hline \multicolumn{1}{|c|}{$\begin{array}{c}\text { Accidentes del } \\
\text { Yrabajo PAÍS }\end{array}$} & $\begin{array}{c}\text { C. No } 121 \text { y/o Parte } \\
\text { VI del C. No } 102\end{array}$ \\
\hline Alemania & $\begin{array}{c}\text { C. } 102(1958) \\
\text { C. } 121(1962)\end{array}$ \\
\hline Grecia & C. $102(1955)$ \\
\hline Irlanda & C. $121(1969)$ \\
\hline Luxemburgo & C. $102(1964)$ \\
\hline Ex República & C. $121(1972)$ \\
\hline Yugoslavia de $102(1991)$ & C. $121(1991)$ \\
\hline Macedonia & C. $102(2006)$ \\
\hline Montenegro & C. $121(2006)$ \\
\hline Países Bajos & C. $102(1962)$ \\
\hline Noruega & C. $121(1966)$ \\
\hline Portugal & C. $102(1966)$ \\
\hline Serbia $102(1994)$ \\
\hline Slovenia & C. $121(2000)$ \\
\hline España & C. $102(1992)$ \\
\hline Suecia & C. $121(1992)$ \\
\hline Suiza & C. $102(1988)$ \\
\hline Turquía & C. $102(1953)$ \\
\hline & C. $121(1969)$ \\
\hline C. $102(1977)$ \\
\hline
\end{tabular}

Fuente: ILOLEX 